\title{
High Predictive Value of Adenosine Provocation in Predicting Atrial Fibrillation Recurrence After Pulmonary Vein Isolation With Visually Guided Laser Balloon Compared With Radiofrequency Ablation
}

\author{
Ekrem Üçer, MD; Sabine Fredersdorf, MD; Joachim Seegers, MD; Florian Poschenrieder, MD; \\ Christian Hauck, MD; Lars Maier, MD; Carsten Jungbauer, MD
}

\begin{abstract}
Background: We recently demonstrated that the acute reconnection rate detected with adenosine provocation test (APT) was significantly lower after pulmonary vein isolation (PVI) with visually guided laser balloon ablation (VGLB) than with RF ablation (RF). We evaluated the recurrence rate of atrial arrhythmias at 12 months after VGLB vs. RF and the significance of APT results for the outcome.

Methods and Results: Fifty patients with paroxysmal AF were randomized to either RF or VGLB ablation in a 1:1 fashion. After PVI each PV underwent an APT. All patients underwent a 3-day Holter and clinical follow-up every 3 months. Significantly less PVs reconnected during APT in the VGLB-arm (10 PV (10.8\%) vs. $29 \mathrm{PV}(30.9 \%) ; \mathrm{P}=0.001)$. Significantly less patients had a recurrence of atrial arrhythmia in the VGLB-arm ( 3 vs. 9; $P=0.047$ ). In the VGLB-arm no recurrence was seen in those patients with a negative APT (negative predictive value (NPV)=100\%). Only 3 of the 8 patients with a positive APT in the VGLB-arm had a recurrence (positive PV (PPV) $=37 \%)$. Recurrences in the RF-arm were seen in 3 patients with positive APT as well as in 6 patients with negative APT (PPV $=18 \%$ and $N P V=33 \%)$.
\end{abstract}

Conclusions: There was significantly less recurrence of atrial arrhythmias at 12 months after PVI with VGLB. A negative APT after PVI with VGBL predicted freedom from AF with a very high NPV meaning that the high acute efficiency of the VGLB persisted long term.

Key Words: Adenosine; Atrial fibrillation; Catheter ablation; Lasers; Recurrence

$\bigcirc$ ince the pioneering study of Haïssaguerre et al, pulmonary vein isolation (PVI) is the cornerstone of atrial fibrillation (AF) ablation procedures. ${ }^{1}$ Unfortunately, in $30-50 \%$ of cases AF recurs despite complete electrical disconnection at the PVs index procedure. The major cause of recurrence is reconnection of the initially isolated PVs. Indeed, $80 \%$ of the patients with recurrence of AF demonstrate at least 1 reconnected PV.,23 A method of detecting the PVs that might reconnect in the future is to administer adenosine during the index procedure and look for the so-called "dormant conduction" ${ }^{4}$ Indeed, after PVI with radiofrequency energy (RF), $17-30 \%$ of isolated PVs in $35-59 \%$ of patients reconnected after APT. ${ }^{5,6}$ Moreover, some studies, including a meta-analysis, indicate a higher rate of $\mathrm{AF}$ recurrence in patients with $\mathrm{PV}$ reconnection after adenosine administration..$^{\mathbf{5}, 6}$ The reconnection of PVs probably depends on the degree of transmural damage during the index procedure, which corresponds to the acute efficiency of the ablation lesion and can be appreciated with adenosine administration.

Point-by-point ablation with RF energy is technically challenging, and achieving a transmural and contiguous ring of necrosis might be difficult. To overcome these problems, balloon catheters using other energy sources such as cryo- or laser-energy have been developed. ${ }^{7,8}$ The visually guided laser balloon ablation (VGLB: HeartLight, CardioFocus Inc., Marlborough, MA, USA) is a unique system by which a compliant balloon occludes the PV ostium and the laser energy can be applied with the help of an endoscope under direct visualization around the PV, thus creating histologically proven deep and concrete ablation lesions. We recently published a randomized acute study in which we showed that the acute reconnection rate after PVI is significantly less in patients treated with VGLB than with RF $(10.8 \%$ vs. $30.9 \%$, respectively, $\mathrm{P}=0.001) .{ }^{9}$ The purpose of the present study was to address the clinical

Received November 6, 2019; revised manuscript received December 19, 2019; accepted December 27, 2019; J-STAGE Advance Publication released online February 5, 2020 Time for primary review: 15 days

Internal Medicine II, Cardiology (E.Ü., S.F., C.H., L.M., C.J.), Radiology (F.P.), University Hospital Regensburg, Regensburg; Klinikum Landshut, Internal Medicine I, Cardiology, Landshut (J.S.), Germany

Mailing address: Ekrem Üçer, MD, University Hospital Regensburg, Internal Medicine II, Cardiology, Franz-Josef-Strauss-Allee 11,93053 Regensburg-Germany. E-mail: ekrem.uecer@ukr.de

ISSN-1346-9843 All rights are reserved to the Japanese Circulation Society. For permissions, please e-mail: cj@j-circ.or.jp 
significance of this remarkable finding: Does this apparently higher acute efficiency of PVI with VGLB affect the clinical outcome in comparison with PVI plus RF?

\section{Methods}

Patients with drug-refractory paroxysmal AF and who provided written informed consent were enrolled in the study. The study complied with the Declaration of Helsinki and the protocol was approved by the ethical commission of the University of Regensburg. Informed consent was given by all patients enrolled.

Inclusion criterion was paroxysmal and symptomatic AF according to the current AF classification criteria. ${ }^{10}$ Exclusion criteria were asthma or known allergy to adenosine; left atrial (LA) thrombus; left atrium $>55 \mathrm{~mm}$; left ventricular ejection fraction (LVEF) $<35 \%$; previous LA ablation for AF; NYHA class IV symptoms; myocardial infarction within the previous 60 days; unstable angina; any history of cardiac valve surgery; uncontrolled bleeding; active infection; severe pulmonary disease; previous cardiac valve surgery.

\section{Study Protocol}

Patients were randomized $1: 1$ to PVI with either VGLB or RF ablation. Either a computed tomography (CT) scan or cardiac magnetic resonance imaging was required before the procedure to obtain the LA anatomy.

\section{Ablation Procedure}

In all patients, LA thrombus was excluded either by transesophageal echocardiography or cardiac CT. The ablation procedure was performed under continued oral anticoagulation with phencoumaron (target INR 2.0-3.0) or with dabigatran $(110 \mathrm{mg}$ b.i.d.) according to institutional standards. The ablation procedure was performed under sedation using continuous infusion of propofol. General anesthesia was used only for patients with sleep apnea syndrome and in those who requested general anesthesia (5 patients). An esophageal temperature probe (Circa S-Cath, Circa Scientific, Englewood, CO, USA) was inserted transorally to continuously monitor esophageal temperature during ablation. If it exceeded $39.5^{\circ} \mathrm{C}$, energy delivery was terminated in both arms. Patients who were not in sinus rhythm at the start of procedure were electrically cardioverted to perform the ablation in sinus rhythm. Cardiac signals were recorded with a standard EP recording system (Labsystem Pro-EP, Boston Scientific, Malborough, MA, USA).
\end{abstract}

\section{RF Ablation}

Venous sheaths were placed in both groins $(2 \times 7 \mathrm{~F}$ sheaths in the left femoral vein; $2 \times 8.5 \mathrm{~F}$ SL0 sheaths in the right femoral vein; St. Jude Medical, Saint Paul, MN, USA). After placing a $6 \mathrm{~F}$ octapolar catheter into the coronary sinus and a $6 \mathrm{~F}$ quadripolar catheter along the His bundle region, a double transseptal puncture was performed using a modified Brockenbrough technique. Unfractionated heparin boluses were administered to maintain activated clotting time between 300 and $350 \mathrm{~s}$.

A circumferential mapping catheter and a $3.5-\mathrm{mm}$ mapping/ablation catheter (LassoNav and Navistar Thermocool SF; Biosense Webster Inc., Diamond Bar, CA, USA) were placed in the left atrium. An electroanatomic map of the left atrium was obtained with the Lasso catheter (CartoFAM Module) and was merged with the CT image of the left atrium (CartoMerge Module). After obtaining the baseline PV potentials with the Lasso catheter, $\mathrm{RF}$ ablation was applied around the PV ostia and at the carina between ipsilateral PVs. The RF energy was titrated from $30 \mathrm{~W}$ at the posterior wall to $40 \mathrm{~W}$ for $30 \mathrm{~s}$ at the anterior wall at a maximal temperature of $43^{\circ} \mathrm{C}$ and a flush rate of $15 \mathrm{~mL} / \mathrm{min}$. Ablation was continued until the disappearance of the PV potentials.

\section{VGLB Ablation}

The only difference in the venous access was the exchange of 1 of the $8.5 \mathrm{~F} \mathrm{SL} 0$ sheaths for a $15 \mathrm{~F}$ steerable sheath. A circular mapping catheter (Lasso Non-Nav, Biosense Webster Inc.) was introduced into each target PV to record baseline PV electrograms and to guide the VGLB catheter into the target vein. The balloon was inflated, aiming for complete occlusion of the PV ostium. The grade of the achieved occlusion was defined as I for complete occlusion of the ostium $\left(360^{\circ}\right)$ or if a less than complete occlusion was achieved, as grade II $\left(270-359^{\circ}\right)$, III $\left(180-269^{\circ}\right)$ or IV $\left(<180^{\circ}\right)$, accordingly. To avoid gaps between ablation lesions, we created laser lesions with a $30-50 \%$ lesion overlap using the adjustable $30^{\circ}$ aiming arc of the laser beam, as suggested by previous preclinical and clinical studies. Another important issue was the titration of the laser power. If we could obtain a very good tissue contact, maximal power ( $12 \mathrm{~W}$ for $20 \mathrm{~s}$ ) was chosen. Applying high laser power in areas without complete occlusion and overlying blood causes overheating of the balloon resulting in balloon disruption. In such regions, lower energy levels must be chosen from $5.5 \mathrm{~W}$ (overlying blood) to maximal $7 \mathrm{~W}$ (overlying but moving blood) for a longer time ( $30 \mathrm{~s})$. If the contact area between 2 ipsilateral PVs (i.e., carina region) was not wide, laser power of $8.5-10 \mathrm{~W}$ for $20 \mathrm{~s}$ was applied.

After the first PVI encircling, the balloon was deflated and the PV potentials were assessed with the circular mapping catheter. If not isolated, we looked for the gap by leaving the circular catheter in the PV, if possible, or we ablated the suspected area of gap.

During ablation of the right-sided PVs phrenic nerve pacing was performed to prevent phrenic nerve injury.

\section{APT}

Each isolated PV underwent an APT at least 20 min after isolation. This waiting period was chosen to exclude early spontaneous recovery and was limited to $40 \mathrm{~min}$ after isolation to standardize the APT timing. After excluding spontaneous recovery of the PV conduction, an adenosine bolus of $18 \mathrm{mg}$ followed by a saline flush was administered through a femoral vein. Intracardiac recordings were continuously monitored. Atrial demand pacing just under the current sinus rhythm was started simultaneously with the adenosine bolus to avoid a sinus arrest that would otherwise lead to underdetection of PV reconnection. Adenosine effect was recognized when at least $1 \mathrm{P}$ wave was blocked, or sinus bradycardia or arrest occurred necessitating atrial pacing. PV reconnection was diagnosed when the circular mapping catheter detected PV potentials in a previously isolated PV. PV reconnection was classified as temporary if the PV potentials disappeared after the effect of adenosine diminished or as permanent if the PV stayed reconnected even after the effect of adenosine had ceased. In the case of temporary reconnection, it was left to the discretion of the physician to perform additional ablations 


\begin{tabular}{lccc|}
\hline Table 1. Patients' Demographics & VGLB & RF & P value \\
& $\mathbf{( n = 2 5 )}$ & $(\mathbf{n = 2 5 )}$ & 0.08 \\
Age, years & $59.7 \pm 10.4$ & $65.3 \pm 11.5$ & 0.77 \\
Male & $13(52)$ & $12(48)$ & 0.70 \\
Duration of AF, years & $12(9-42)$ & $16(8-67)$ & 0.49 \\
Hypertension & $21(84)$ & $19(76)$ & 0.73 \\
Diabetes mellitus & $6(24)$ & $5(20)$ & 0.75 \\
Coronary artery disease & $6(24)$ & $7(28)$ & 1.0 \\
Myocardial infarction & $4(16)$ & $4(16)$ & 0.50 \\
Coronary artery bypass grafting & $0(0)$ & $2(8)$ & 1.0 \\
Congestive heart failure & $4(16)$ & $3(12)$ & 1.0 \\
Stroke or transient ischemic attack & $3(12)$ & $4(16)$ & 0.90 \\
Body mass index, m/kg & $28(25-36)$ & $28(25-30)$ & 0.15 \\
Left atrial diameter, mm & $41.3 \pm 5.1$ & $44.8 \pm 7.6$ & 0.83 \\
Ejection fraction, \% & $60.9 \pm 3.8$ & $60.6 \pm 5.1$ & 1.0 \\
Atrial flutter ablation & $3(12)$ & $3(12)$ & 0.47 \\
Antiarrhythmic medications (Class I or III) & $10(40)$ & $8(32)$ & 0.13 \\
EHRA 1/2/3/4 & $1(4) / 5(20) / 13(52) / 6(24)$ & $0 / 12(48) / 7(28) / 6(24)$ & \\
\hline
\end{tabular}

Values are mean $\pm S D, n(\%)$, or median (interquartile range). $A F$, atrial fibrillation; $R F$, radiofrequency; VGLB, visually guided laser balloon.

\begin{tabular}{|c|c|c|c|}
\hline & $\begin{array}{l}\text { VGLB } \\
(n=25)\end{array}$ & $\begin{array}{c}R F \\
(n=25)\end{array}$ & $P$ value \\
\hline Procedure time, $\min ^{\star}$ & $232 \pm 38$ & $237 \pm 60$ & 0.70 \\
\hline Ablation time, $\min ^{\star *}$ & $157 \pm 34$ & $177 \pm 49$ & 0.11 \\
\hline Fluoroscopy time, min & $30(22-36.5)$ & $11(7.5-14.5)$ & $<0.001$ \\
\hline Fluoroscopy dose, cGym² & $1,849(1,504-3,074)$ & $1,114(645-1,755)$ & 0.002 \\
\hline
\end{tabular}

Values are mean $\pm \mathrm{SD}$, or median (interquartile range). ${ }^{*}$ Defined as time from venous access to sheath removal. ${ }^{*}$ Defined as time from the end of the second transseptal puncture to sheath removal. RF, radiofrequency; VGLB, visually guided laser balloon.

and to test the PV again with APT.

\section{Clinical Follow-up}

A blanking period of 90 days was applied. All patients attended outpatient clinical visits for cardiac rhythm disturbances, including 72-h Holter ECG at 3, 6 and 12 months. A recurrence was defined as a documented atrial tachyarrhythmia episode lasting $>30 \mathrm{~s}$.

\section{Re-ablation Procedure}

In the case of symptomatic AF recurrence, we performed a second procedure. According to the recommendations in the expert consensus statement on catheter ablation of AF, our first step in re-ablation procedures was controlling the PVs for possible reconnection. In the case of PV reconnection, our strategy was confined to re-isolate the reconnected PVs. ${ }^{2}$ In the case of sustained PV isolation our strategy incorporated ablation of fragmented potentials, creating of lineal lesion sets or searching for non-PV foci. But these additional ablation concepts were only performed in patients who did not show any reconnected PV in the second procedure. ${ }^{2}$

\section{Statistical Analysis}

Values are distributed as mean \pm SD for normally distrib- uted continuous variables, median and interquartile range (IQR) for skewed distributions (assessed by means of Kolmogorov-Smirnow 1-sample test) and counts and percentages for categorical variables. Statistical analysis was conducted using Student's t-test (unpaired) for continuous variables with normal distribution and Mann-Whitney U test for variables with non-normally distribution. The chi-square test or Fisher's exact test was used to compare the categorical variables in different arms. Statistical significance was defined as $\mathrm{P}<0.05$. Statistical analysis was performed using SPSS 21 (SPSS Inc., Chicago, IL, USA).

\section{Results}

A total of 50 patients were randomized 1:1 into the VGLB or RF ablation groups. All patients had paroxysmal AF.

\section{Patients and Procedural Characteristics}

There were no significant differences in basic clinical characteristics between the 2 arms (Table 1).

Although total and LA procedure times were equal in both arms, fluoroscopy times were significantly shorter in the RF-arm (Table 2).

A total of 3 patients in the VGLB-arm and 4 in the RF-arm had a common PV ostium, thus 97 PVs in the 


\begin{tabular}{llcc|}
\hline \multicolumn{1}{l}{ Table 3. Comparison of Reconnected and Non-Reconnected PVs in VGLB-Arm } \\
& $\begin{array}{c}\text { APT-negative } \\
(\mathbf{n}=\mathbf{8 3})\end{array}$ & $\begin{array}{c}\text { APT-positive } \\
(\mathbf{n = 1 0 )}\end{array}$ & P value \\
Time to APT, min* & $28(22.0-36)$ & $29(23.5-37.5)$ & 0.71 \\
Application time, s & $570(454-785)$ & $955(485-1,053)$ & 0.19 \\
No. of applications & $29(24-39)$ & $46.5(25-52)$ & 0.22 \\
Mean power, W & $10 \pm 1.0$ & $8.8 \pm 1.5$ & 0.01 \\
Total energy, J & $5,720(4,490-7,232)$ & $7,952(4,996-10,272)$ & 0.16 \\
Occlusion grade & $1(1-1)$ & $1.5(1-4)$ & 0.002 \\
\hline
\end{tabular}

Values are mean $\pm \mathrm{SD}$ or median (interquartile range). ${ }^{*}$ Defined as time from the isolation of a vein to the administration of adenosine for that vein. APT, adenosine provocation test; VGLB, visually guided laser balloon.

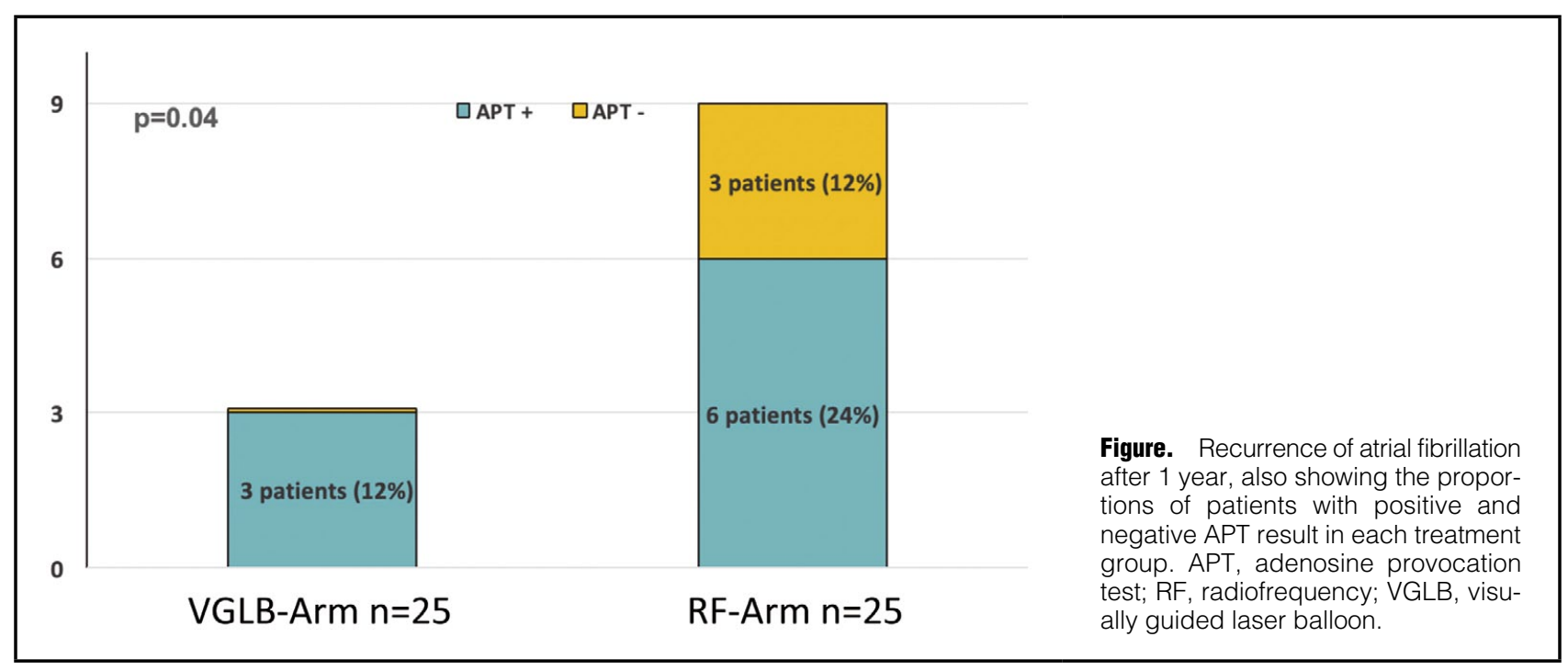

VGLB-arm and 96 in the RF-arm were targeted for PVI; 93 PVs $(95.9 \%)$ in the VGLB-arm and $94(97.9 \%)$ in the $\mathrm{RF}$-arm were successfully isolated $(\mathrm{P}=0.41)$. The right inferior PVs could not be isolated in 2 patients in each arm because of temperature exceeding $39.5^{\circ} \mathrm{C}$ in the esophagus. Additionally, in 1 patient the left common ostium and in another patient the right inferior PV could not be isolated in the VGLB-arm because of temperature rise in the esophagus and as well as incomplete occlusion of the veins with the balloon resulting in ablation with low laser energy.

Significantly more PVs were isolated after the first encirclement in the VGLB-arm than in the RF-arm $(80.4 \%$ (78 PVs) vs. 47.9\% (46 PVs), respectively; $\mathrm{P}<0.001$ ).

\section{APT}

All isolated PVs underwent an APT. Mean time to APT after isolation of each PV was not different in both therapy arms (28 $\mathrm{min}$ in VGLB vs. $31.5 \mathrm{~min}$ in $\mathrm{RF}$; $\mathrm{P}=0.12)$. In the VGLB group significantly less PVs had a positive APT than in the RF group (10 (10.8\%) vs. $29(30.9 \%), \mathrm{P}=0.001)$. Of all the reconnected PVs, only 1 in the VGLB-arm and 2 in the RF-arm had a permanent reconnection. More patients had at least $1 \mathrm{PV}$ with reconnection in the RF-arm than in the VGLB-arm $(n=16(64 \%)$ vs. $n=8(32 \%), P=0.02)$. In the VGLB-arm reconnection under adenosine was seen in 3 of the left superior (LS) PV, in 4 of the left inferior (LI) $\mathrm{PV}$, in 1 of the right superior (RS) PV and in 2 of the right inferior (RI) PV; in the RF-arm in 7 of the LSPV, in 5 of the LIPV, in 1 of the left common PV, in 8 of the RSPV and in 8 of the RIPV. There was significantly less reconnection in the RSPV after PVI with VGLB than with RF $(\mathrm{P}=0.023)$. There was also a strong trend to less reconnection in the RIPV after PVI with VGLB. There was no difference in the adenosine administration time when PVs with reconnection were compared with PVs without reconnection in each therapy arm separately.

\section{Differences in Clinical and Procedural Parameters in the APT-Positive and -Negative Arms}

The clinical characteristics of patients with or without reconnection did not differ between therapy arms (each $\mathrm{P}=\mathrm{NS}$ ). Only a minority of patients had general anesthesia (5 patients, 10\%). The type of anesthesia had no influence on the reconnection rates; $4(20 \%)$ of $20 \mathrm{PVs}$ had a positive APT in patients with general anesthesia vs. $35(21 \%)$ of 167 PVs in patients with deep sedation, $\mathrm{P}=1.0$. PVs with reconnection were more likely to be incompletely occluded with the laser balloon (balloon occlusion grade 1.5 vs. 1.0, respectively; $\mathrm{P}=0.002)$ and to be ablated with less power $(8.8 \pm 1.5 \mathrm{~W}$ to $10 \pm 1.0 \mathrm{~W}$, respectively; $\mathrm{P}=0.01)$ (Table 3 ). No significant differences in the ablation data (total energy applied $250.6 \pm 67.1 \mathrm{~kJ}$ vs. $227.9 \pm 67.8 \mathrm{~kJ}, \mathrm{P}=0.42$; ablation time $3,611 \pm 1,029 \mathrm{~s}$ vs. $3,152 \pm 865 \mathrm{~s} ; \mathrm{P}=0.27)$ and the time to ATP (31 min vs. $34 \mathrm{~min} ; \mathrm{P}=0.0194)$ could be detected in the $\mathrm{RF}$-arm between reconnected and non-reconnected PVs. 


\section{Re-ablation After Positive APT}

A total of 3 PVs (1 with permanent reconnection) in the VGLB-arm and 14 PVs (2 with permanent reconnection) in the RF-arm were re-ablated because of positive APT. Because we did not use a 3D mapping system with VGLB the exact reconnection site was more difficult to localize than in patients undergoing RF ablation. Nevertheless, using the shaft position of the Lasso catheter under fluoroscopy was very helpful for localizing the sector (i.e., Lasso in LSPV and shaft in the superior position on fluoroscopy, then electrodes 5-6 point to the posterior-inferior segment of the PV) where the conduction gap could be found and successfully ablated. At $20 \mathrm{~min}$ after re-isolation, a second APT was performed. In 2 of the 3 re-ablated PVs in the VGLB-arm and in 6 of the 14 in the RF-arm no reconnection was detected with repeat APT. The PVs with permanent reconnection did not reconnect after re-isolation with the second APT.

\section{Clinical Follow-up}

Each patient underwent a clinical visit with Holter ECG quarterly during the 1-year follow-up period. Significantly less patients had a recurrence of atrial arrhythmias in the VGLB-arm at 12 months ( 3 vs. 9; $P=0.047$ ). In the VGLBarm 21 patients were completely free of any complaints and there were not any atrial arrhythmias on Holter ECG. One patient described palpitations, which were attributed to newly diagnosed ventricular premature beats. In 3 patients of the VGLB-arm, paroxysmal AF recurred, which was also detected on Holter ECG. In the RF-arm 9 patients had a recurrence, 2 of them with asymptomatic episodes detected on routine Holter ECG. In another 3 patients, repeated Holter ECGs had to be performed to confirm the recurrence of AF according to the complaints of the patients. In 2 patients, an atypical atrial flutter was also detected in addition to paroxysmal AF episodes (Figure).

\section{Correlation of the APT Results With AF Recurrence}

Interestingly, no recurrence was seen in any of the patients with a negative APT in the VGLB-arm. Only 3 of the 8 patients with a positive APT in the VGLB-arm had a recurrence. None of the patients in the VGLB-arm had a recurrence if re-ablated because of positive APT after initial isolation.

Recurrences in the RF-arm were seen in 3 of 16 patients with positive APT as well as in 6 of 9 patients with negative APT (Figure). Sensitivity of the APT in predicting the atrial arrhythmias in the VGLB-arm was $100 \%$ with a specificity of $85 \%$, whereas in the RF-arm sensitivity was $33 \%$ and specificity $18 \%$. Accordingly, the negative predictive value (NPV) of the APT test in the VGLB-arm was $100 \%$ and the positive predictive value (PPV) was $37 \%$; in the RF-arm the NPV and PPV was $33 \%$ and $18 \%$, respectively.

In the VGLB-arm none of the patients re-ablated because of a positive APT (who could also be changed to have completely negative APT) had a recurrence; 1 of the 3 patients with recurrence in the APT-positive RF-arm was not re-ablated and the other 2 were re-ablated but could not be converted to a completely negative APT state.

\section{Findings From the Re-ablation Procedure}

Re-ablation was performed in 2 of the 3 patients with recurrence of $\mathrm{AF}$ in the VLGB-arm and in 6 of the 9 patients in the RF-arm; 2 of the other 4 patients had only asymptomatic recurrences, so re-ablation was not offered, and the other 2 patients did not want to have a second procedure. All repeat procedures were performed with the help of a 3D mapping system and RF energy was used. The 2 patients in the VLGB-arm with recurrence had a temporary positive APT in the initial procedure and interestingly the same PVs were reconnected in the second procedure (both left PVs in 1 and the LSPV in the other patient). Of the 6 re-ablated patients in the RF-arm 4 had negative APT for all PVs in the initial procedure; in these patients 7 PVs had reconnected. In the other 2 patients with reconnection in the initial procedure all of the PVs had reconnected. Re-isolation was achieved with point ablations with RF energy around the PVs in all these patients.

Because all of our patients with AF recurrence had at least one or more reconnected PVs there was no need to perform further ablations such as lines or ablation of complex fragmented signals or non-pulmonary foci. There were no procedure-related complications during the second procedure in patients with recurrence.

All of the patients who were re-ablated were free of symptoms in the following 6 months.

\section{Complications}

In total, 3 patients in the VGLB-arm and 1 patient in the RF-arm developed a slight groin hematoma, which was treated conservatively $(\mathrm{P}=\mathrm{NS})$. In 1 female patient, pericardial tamponade occurred during the placement of the diagnostic catheter into the coronary sinus, but 4 weeks later a successful PVI with RF was performed. This complication was classified as procedure- but not device-related complication. In 1 male patient, the atrial septal defect after VGLB persisted, so it had to be closed with an atrial septum closure device 2 months after the procedure.

\section{Discussion}

The first important finding in our study was that the higher acute efficiency of VGLB, which has been shown previously, appeared to be clinically persistent after 1 year. None of the patients in the VGLB-arm in whom the APT was negative for all of the PVs had recurrence of AF. Interestingly, patients with recurrence in the VGLB-arm, who were re-ablated, showed reconnection in those PVs that had showed temporary reconnection in the initial procedure. These data imply that effective VGLB ablation without dormant connection in the initial ablation procedure leads to a clinically successful outcome without recurrence of AF after 1 year. On the other hand, findings in the RF-arm were not easy to interpret because the results of the APT during the initial ablation had very poor predictive value for clinical success. According to the results of our study, having no reconnection with APT during the initial procedure in the RF-arm did not mean that there would be no recurrence of AF during follow-up. These findings are in accordance with 2 important trials in which the dormant connection after PVI with adenosine was tested: the ADVICE and the UNDER-ATP studies. ${ }^{11,12}$ It is well known that there is a higher probability of developing AF recurrence if $\geq 1 \mathrm{PVs}$ reconnect during adenosine, but further ablation of the reconnected PVs does not result in better outcomes (i.e., in the Under-ATP trial, ${ }^{6,12}$ Gula et al showed that a positive adenosine test had a high PPV $(90 \%)$ in predicting recurrence whereas a negative test had very low predictive value (15\%) after PVI with RF energy ${ }^{13}$ ). 
Our results are somewhat in line with that study, as we had a very poor NPV (18\%) but also a poor PPV $(33 \%)$ in the RF-arm. Interestingly, this was not the case for the VGLBarm, in which a NPV of $100 \%$ was achieved. The reason for that might be, as shown previously, in the different characteristics of the ablation lesions created with various techniques. Gerstenfeld et a ${ }^{14}$ analyzed the lesions created with laser and with RF energy in a swine model. Histopathological examinations showed that the lesions created with laser energy were all circumferential and transmural whereas none of the lesions by RF were transmural. Moreover, only those PVs with transmural lesions after VGLB ablation remained persistently isolated, whereas all PVs in the RF-arm were reconnected and all of them had non-transmural lesions. ${ }^{14}$ Dukkipati et al performed 2 studies in which the patients underwent a second LA procedure to check the PVs at 3 months after the index VGLB procedure $86-90 \%$ of the PVs were still disconnected. ${ }^{\mathbf{1 5}, 16}$ In contrast, a similar remapping study after PVI with RF showed a disconnection rate of only $57 \% .^{17}$ Accordingly, we can assume that VGLB ablation has the potential to create true transmural lesions, which might be reflected by the higher acute efficiency detected by APT. Additionally, this leads to a higher rate of persistence of PVI with a better clinical outcome. Thus, developing a strategy to isolate the PVs with VGLB in the first procedure until APT shows no more reconnection could lead to better outcomes (i.e., in patients with paroxysmal AF; especially for those patients who have their triggers in the PVs). Interestingly, re-isolation of the reconnected PVs was sufficient in patients with symptomatic AF recurrence in the current study, as they had no symptomatic recurrences in the following 6 months. The expert consensus for catheter ablation of AF also suggests re-isolation of PVs as the first step, if there are any reconnected PVs in the second procedure after AF recurrence, especially in paroxysmal AF. ${ }^{2}$ Although the possibility of having non-PV foci in some patients cannot be excluded generally, re-isolation of reconnected PVs as the first step in treating paroxysmal $\mathrm{AF}$ is an effective strategy before creating more aggressive ablation lesions. ${ }^{2}$ The finding that none of our patients had recurrence after the re-isolation procedure shows again that the PVs are the major cause of AF and also the importance of achieving effective PV isolation during the first procedure.

The noninferiority of VGLB ablation had already been shown in paroxysmal AF patients in a multicenter trial, but a provocation test was not performed in that trial. ${ }^{18}$ The question of whether an APT would have affected the result of this study cannot be answered, but aiming to create lesion sets that are very robust seems to be effective in terms of clinical outcome, even with RF energy, as shown recently by Phlips et a ${ }^{19}$ in the Close to Cure trial. They showed that a strict ablation strategy (with contact force measurement using ablation index criteria and maximal interlesion distance of $\leq 6 \mathrm{~mm}$ ) aiming for durable isolation of the PVs resulted in a major overall reduction in the atrial tachyarrhythmia burden. The aim of this ablation strategy was to examine the effect of enclosing the PV ostia with contiguous, stable and optimized RF lesions on the clinical outcome. Indeed, a "nearly" perfect PV isolation resulted in freedom of atrial tachyarrhythmias as detected in $88 \%$ of patients with paroxysmal AF after 1 year. ${ }^{19}$ These data suggest again the importance of durable PVI, especially in patients with paroxysmal AF.

\section{Study Limitations}

One of the limitations of our study might be the low number of patients. The largest randomized study that compared the clinical efficiency of VGBL to RF had 178 patients in the VGLB-arm and 175 in the RF-arm and showed the noninferiority of the VGLB. ${ }^{18}$ But that study did not include APT as part of the study protocol. We believe that the fact that none of the patients $(0 \%)$ with negative APT in the VGLB-arm had a recurrence is a very impressive finding. Because of the very high NPV of APT, even small studies that compare the clinical outcomes after PVI with VGLB and RF might show significant differences if an APT was included in the study protocol.

The second major limitation is that we did not use contact force ablation catheters in the RF-arm, which could affect the study results. This tool could have led to more effective RF ablation and thus influence the study results, because the reconnection rate with contact force catheters is lower than with the standard RF catheters $(8 \%$ vs. $35 \%){ }^{20}$ Nevertheless, the main finding of the present study, which is the very high NPV of APT in VGBL only, would not have been affected if we had used RF ablation with contact force catheters and ablation index data. However, further studies should be performed with new RF ablation strategies using contact force measurement, ablation index and high power/short duration and with the new laser balloon with automatically rotating motor, which has been recently launched. According to our data, we believe that an APT should always be included in studies dealing with clinical outcomes after PVI using different technologies.

Information about the exact regions of the reconnections in each PV is lacking, as well as a comparison of these regions between the 2 ablation systems. Such data would help clinicians be aware of the critical regions for reconnection, thus more efforts would be performed in gaining effective lesions in such regions, depending on the applied energy.

\section{Conclusions}

A negative APT after PVI with VGBL predicted freedom from AF with a very high NPV, meaning that the high acute efficiency of the VGLB persisted in the long term. Further studies should be conducted with adequate power to detect differences in the outcome after adenosine-guided PVI with next-generation VGLB and new ablation strategies using RF.

\section{References}

1. Haïssaguerre M, Jaïs P, Shah DC, Takahashi A, Hocini M, Quiniou G, et al. Spontaneous initiation of atrial fibrillation by ectopic beats originating in the pulmonary veins. $N$ Engl J Med 1998; 339: 659-666.

2. Calkins H, Hindricks G, Cappato R, Kim YH, Saad EB, Aguinaga L, et al. $2017 \mathrm{HRS} / \mathrm{EHRA} / \mathrm{ECAS} / \mathrm{APHRS} / \mathrm{SOLAECE}$ expert consensus statement on catheter and surgical ablation of atrial fibrillation. Europace 2018; 20: e1-e160.

3. Medi C, Sparks PB, Morton JB, Kistler PM, Halloran K, Rosso $\mathrm{R}$, et al. Pulmonary vein antral isolation for paroxysmal atrial fibrillation: Results from long-term follow-up. $J$ Cardiovasc Electrophysiol 2011; 22: 137-141.

4. Datino T, Macle L, Qi XY, Maguy A, Comtois P, Chartier D, et al. Mechanisms by which adenosine restores conduction in dormant canine pulmonary veins. Circulation 2010; 121: 963-972.

5. Arentz T, Macle L, Kalusche D, Hocini M, Jais P, Shah D, et al. "Dormant" pulmonary vein conduction revealed by adenosine after ostial radiofrequency catheter ablation. J Cardiovasc Electrophysiol 2004; 15: 1041 - 1047. 
6. McLellan AJ, Kumar S, Smith C, Morton JB, Kalman JM, Kistler PM. The role of adenosine following pulmonary vein isolation in patients undergoing catheter ablation for atrial fibrillation: A systematic review. J Cardiovasc Electrophysiol 2013; 24: $742-751$.

7. Packer DL, Kowal RC, Wheelan KR, Irwin JM, Champagne J, Guerra PG, et al. Cryoballoon ablation of pulmonary veins for paroxysmal atrial fibrillation: First results of the North American Arctic Front (STOP AF) pivotal trial. J Am Coll Cardiol 2013; 61: $1713-1723$.

8. Dukkipati SR, Kuck KH, Neuzil P, Woollett I, Kautzner J, McElderry HT, et al. Pulmonary vein isolation using a visually guided laser balloon catheter: The first 200 patient multicenter clinical experience. Circ Arrhythm Electrophysiol 2013; 6: 467-472.

9. Ücer E, Janeczko Y, Seegers J, Fredersdorf S, Friemel S, Poschenrieder F, et al. A RAndomized Trial to compare the acute reconnection after pulmonary vein ISolation with Laser-BalloON versus radiofrequency Ablation: RATISBONA trial. J Cardiovasc Electrophysiol 2018; 29: 733-739.

10. Kirchhof P, Benussi S, Kotecha D, Ahlsson A, Atar D, Casadei B, et al. 2016 ESC Guidelines for the management of atrial fibrillation developed in collaboration with EACTS. Europace 2016; 18: 1609-1678.

11. Macle L, Khairy P, Weerasooriya R, Novak P, Verma A, Willems $\mathrm{S}$, et al. Adenosine-guided pulmonary vein isolation for the treatment of paroxysmal atrial fibrillation: An international, multicentre, randomized superiority trial. Lancet 2015; 386: 672 679 .

12. Kobori A, Shizuta S, Inoue K, Kaitani K, Morimoto T, Nakazawa Y, et al; UNDER-ATP Trial Investigators. Adenosinetriphosphateguided pulmonary vein isolation for atrial fibrillation: The Unmasking Dormant Electrical Reconduction by Adenosine TriPhosphate (UNDER-ATP) trial. Eur Heart J 2015; 36: 32763287.

13. Gula LJ, Massel D, Leong-Sit P, Gray C, Fox DJ, Segal QR, et al. Does adenosine response predict clinical recurrence of atrial fibrillation after pulmonary vein isolation? J Cardiovasc Electrophysiol 2011; 22: 982-986.

14. Gerstenfeld E, Michele J. Pulmonary vein isolation using a compliant endoscopic laser balloon ablation system in a swine model. J Interv Card Electrophysiol 2010; 29: 1-9.

15. Dukkipati SR, Neuzil P, Skoda J, Petru J, d'Avilla A, Doshi SK, et al. Visual balloon-guided point-by-point ablation: Reliable, reproducible, and persistent pulmonary vein isolation. Circ Arrhythm Electrophysiol 2010; 3: 266-273.

16. Dukkipati SR, Neuzil P, Kautzner J, Petru J, Wichterle D, Skoda J, et al. The durability of pulmonary vein isolation using the visually guided laser balloon catheter: Multicenter results of pulmonary vein remapping studies. Heart Rhythm 2012; 9: 919925.

17. Willems S, Steven D, Servatius H, Hoffmann BA, Drewitz I, Müllerleile K, et al. Persistence of pulmonary vein isolation after robotic remote-navigated ablation for atrial fibrillation and its relation to clinical outcome. J Cardiovasc Electrophysiol 2010; 21: $1079-1084$.

18. Dukkipati SR, Cuoco F, Kutinsky I, Aryana A, Bahnson TD, Lakkireddy D, et al. Pulmonary vein isolation using the visually guided laser balloon: A prospective, multicenter, and randomized comparison to standard radiofrequency ablation. $\mathrm{J} \mathrm{Am}$ Coll Cardiol 2015; 66: 1350-1360.

19. Phlips T, Taghji P, El Haddad M, Wolf M, Knecht S, Vandekerckhove Y, et al. Improving procedural and one-year outcome after contact force-guided pulmonary vein isolation: The role of interlesion distance, ablation index, and contact force variability in the 'CLOSE'-protocol. Europace 2018; 20: f419f427.

20. Andrade JG, Monir G, Pollak SJ, Khairy P, Dubuc M, Roy D, et al. Pulmonary vein isolation using "contact force" ablation: The effect on dormant conduction and long-term freedom from recurrent atrial fibrillation - a prospective study. Heart Rhythm 2014; 11: 1919-1924. 